\title{
Esophageal Capsule Endoscopy for Screening and Surveillance of Esophageal Varices in Patients with Portal Hypertension
}

\author{
Roberto de Franchis, ${ }^{1}$ Glenn M. Eisen, ${ }^{2}$ Loren Laine,${ }^{3}$ Inaki Fernandez-Urien, ${ }^{4}$ Juan Manuel Herrerias, ${ }^{5}$ \\ Russell D. Brown, ${ }^{6}$ Laurel Fisher, ${ }^{7}$ Hugo E. Vargas,${ }^{8}$ John Vargo,${ }^{9}$ Julie Thompson, ${ }^{10}$ and Rami Eliakim ${ }^{11}$
}

Bleeding from esophageal varices (EV) is a serious consequence of portal hypertension. Current guidelines recommend screening patients with cirrhosis with esophagogastroduodenoscopy (EGD) to detect varices. However, the unpleasantness and need for sedation of EGD may limit adherence to screening programs. Pilot studies have shown good performance of esophageal capsule endoscopy in detecting varices. This multicenter trial was designed to assess the diagnostic performance of capsule endoscopy in comparison with EGD. Patients undergoing EGD for screening or surveillance of EV underwent a capsule study previously. The study was designed as an equivalence study, assuming that a difference of $\leq 10 \%$ between capsule endoscopy and EGD in diagnosing EV would demonstrate equivalence. Two hundred eighty-eight patients were enrolled. Endoscopy was for screening in 195 patients and for surveillance of known EV in 93. Overall agreement for detecting EV between EGD and capsule endoscopy was 85.8\%; the kappa score was 0.73. Capsule endoscopy had a sensitivity, specificity, positive predictive value, and negative predictive value of $84 \%, 88 \%, 92 \%$, and $77 \%$, respectively. The difference in diagnosing EV was $15.6 \%$ in favor of EGD. There was complete agreement on variceal grade in 227 of 288 cases (79\%). In differentiating between medium/large varices requiring treatment and small/ absent varices requiring surveillance, the sensitivity, specificity, positive predictive value, and negative predictive value for capsule endoscopy were $78 \%, 96 \%, 87 \%$, and $92 \%$, respectively. Overall agreement on treatment decisions based on EV size was substantial at $91 \%$ (kappa = 0.77). Conclusion: We recommend that EGD be used to screen patients with cirrhosis for large EV. However, the minimal invasiveness, good tolerance, and good agreement of capsule endoscopy with EGD might increase adherence to screening programs. Whether this is the case needs to be determined. (HePATOLOGY 2008;47:1595-1603.)

See Editorial on Page 1434

T he average risk of variceal bleeding in unselected populations of patients with cirrhosis with esophageal varices is about $25 \% .{ }^{1}$ Such bleeding carries a mortality of $10 \%-20 \%$ within 6 weeks of the bleeding episode. ${ }^{2-4}$ The risk of bleeding is related to the size of varices, the presence of "red signs" on varices, and the degree of liver insufficiency as evaluated by the ChildPugh score. ${ }^{5}$

Because nonselective beta blockers and band ligation decrease the relative risk of bleeding by about $50 \%$ in patients with medium or large varices, ${ }^{1,6}$ practice guide-

Abbreviations: CI, confidence interval; EGD, esophagogastroduodenoscopy; EV, esophageal varices; HBV, hepatitis $B$ virus; HCV, hepatitis $C$ virus; PHG, portal hypertensive gastropathy; PVT, portal vein thrombosis.

From the ${ }^{I}$ Department of Medical Sciences, University of Milan, Istituto di Ricovero e Cura a Carattere Scientifico Fondazione Ospedale Maggiore Policlinico, Mangiagalli and Regina Elena, Milan, Italy; ${ }^{2}$ Oregon Health Sciences University, Portland, OR; ${ }^{3}$ University of Southern California, Los Angeles, CA; ${ }^{4}$ Universidad de Navarra, Pamplona, Spain; ${ }^{5}$ Hospital Universitario Virgen Macarena, Seville, Spain; ${ }^{6}$ University of Illinois at Chicago, Chicago, IL; ${ }^{7}$ University of Michigan, Ann Arbor, MI; ${ }^{8}$ Mayo Clinic Foundation, Scottsdale, AZ; ${ }^{9}$ Cleveland Clinic Foundation, Cleveland, OH; ${ }^{10}$ University of Minnesota, Minneapolis, MN; and ${ }^{11}$ Rambam Medical Center, Haifa, Israel.

Received August 6, 2007; accepted January 9, 2008.

Address reprint requests to: Roberto de Franchis, Department of Medical Sciences, University of Milan, Istituto di Ricovero e Cura a Carattere Scientifico Fondazione

Ospedale Maggiore Policlinico, Mangiagalli and Regina Elena, Milan, Italy. E-mail: roberto.defranchis@unimi.it; fax: (39)025 0320747.

Copyright (C) 2008 by the American Association for the Study of Liver Diseases.

Published online in Wiley InterScience (www.interscience.wiley.com).

DOI 10.1002/hep.22227

Potential conflict of interest: Drs. de Franchis, Eliakim, and Eisen are consultants for and received grants from Given Imaging. Dr. Laine received grants from Given Imaging. Dr. Brown is on the speakers' bureau of Given Imaging. Dr. Fischer is a consultant for and is on the speakers' bureau of Given Imaging. Dr. Vargas received grants from Roche, Novartis, Vertex, Idenix, Pharmasset, Debiopharm, and Given Imaging. 
lines $^{7-10}$ for the treatment of portal hypertension recommend that all patients should undergo endoscopic screening for varices at the time when cirrhosis is diagnosed. After screening endoscopy, patients with medium or large varices should be treated to prevent bleeding, whereas all other patients should undergo periodic surveillance endoscopy. The recommended intervals are 2-3 years for patients with compensated disease and no varices, 1-2 years for those with small varices, ${ }^{11}$ and 1 year for those with decompensated disease, with or without varices. ${ }^{8,11}$

These recommendations generate a considerable burden in endoscopies and related costs. In addition, they require that patients repeatedly undergo a procedure that is perceived as unpleasant, requires conscious sedation in most cases, may lead to decreased work productivity, and has a small but not insignificant risk of complications, ${ }^{12}$ even though up to $50 \%$ of patients may still not have developed esophageal varices 10 years after the diagnosis of cirrhosis. ${ }^{13,14}$ These factors may decrease patient compliance, leading to a decrease in the effectiveness of the screening program.

In recent years, a number of studies ${ }^{15}$ have addressed the issue of identifying patients with varices by noninvasive means, with the aim of avoiding endoscopy in those at low risk of having varices. These studies have assessed the potential of biochemical, clinical, and ultrasound parameters, of blood markers of fibrosis, of transient elastography, and of multidetector computed tomography esophagography for the noninvasive (or minimally invasive) diagnosis of the presence of esophageal varices.

The esophageal videocapsule endoscopy system (PillCam ESO, Given Imaging, Ltd., Yoqneam, Israel) provides another less invasive approach to visualizing the esophagus. Advantages include the elimination of the need for conscious sedation, the minimally invasive nature of the test, and the ability to pursue normal daily activities following the procedure. Furthermore, capsule endoscopy is likely to be more readily accepted by patients than standard esophagogastroduodenoscopy (EGD). A previous study with the esophageal video capsule showed accurate detection of findings such as erosive esophagitis and Barrett's esophagus. ${ }^{16}$ In addition, three pilot studies ${ }^{17-19}$ in patients with cirrhosis suggest that the esophageal capsule may be useful in the detection and assessment of the size of esophageal varices.

The primary aim of the present multicenter study was to assess the accuracy of the esophageal capsule in identifying the presence of esophageal varices in patients undergoing screening or surveillance for esophageal varices by EGD. Secondary objectives were (1) to assess the accuracy of the esophageal capsule in grading esophageal varices compared to EGD; (2) to evaluate the accuracy of the esophageal capsule in differentiating medium/large esophageal varices, which require treatment, from small or absent varices, which require surveillance; and (3) to assess the preprocedure patient perception of and postprocedure patient satisfaction with the esophageal capsule compared to standard EGD.

\section{Patients and Methods}

This is a multicenter prospective study carried out in 11 centers in the United States, Europe, and Israel.

\section{Inclusion Criteria}

- Age 18 years or older.

- Signs/symptoms of portal hypertension, without previous diagnosis of esophageal varices, with clinical indication for screening endoscopy for the detection of varices, or with prior endoscopic diagnosis of esophageal varices and indication for surveillance endoscopy.

- Signed informed consent form.

\section{Exclusion Criteria}

- Dysphagia.

- Known Zenker's diverticulum.

- Previous endoscopic treatment of esophageal varices.

- Known or suspected intestinal obstruction.

- Cardiac pacemakers or other implanted electromedical devices.

- Pregnancy.

- Planned magnetic resonance imaging examination within 7 days after ingestion of the capsule.

- Prior abdominal surgery of the gastrointestinal tract (other than uncomplicated appendectomy or uncomplicated cholecystectomy).

- Any condition that precludes compliance with study and/or device instructions.

- Life-threatening conditions.

- Current participation in another clinical study.

\section{Study Procedures}

Esophageal Capsule Endoscopy. The capsule examination was performed prior to sedation and EGD in all patients. After fasting for at least 5 hours, the patients swallowed the capsule, following the standard procedure. In short, the patients were asked to swiftly drink $100 \mathrm{~mL}$ of water to clear saliva and were positioned flat on the bed with their head on a pillow. The capsule was ingested with a minimum amount of water, without the head being raised from the pillow. The patients remained in the su- 
pine position for 2 minutes, after which the bed was raised to an angle of 30 degrees for 2 minutes more and then to 60 degrees for 1 minute. At the end of this period, the patients were free to sit or walk for the next 15 minutes, but without drinking or eating until the procedure ended. The total duration of the procedure was 20 minutes. ${ }^{15}$

The videos were reviewed and graded by gastroenterologists who were experienced in capsule endoscopy and were blinded to the results of endoscopic examinations. They were instructed to grade the varices according to the grading system described in the Study Measurements section and to record the presence of portal hypertensive gastropathy (PHG). No formal evaluation of gastric varices was carried out because the capsule was considered unsuitable for gastric varices. No specific instructions on how to read the capsule videos (for example, the speed of viewing) were given.

Separate investigators read the capsule videos and performed the conventional EGD examination.

Upper GI Endoscopy. EGD was performed under light sedation within 48 hours after the capsule procedure. The endoscopist was not blinded to the patient's prior medical history but was blinded to the preceding capsule results. In each EGD, the investigator captured a minimum of 4 photographs including the esophageal body, squamocolumnar junction, diaphragmatic pinchcock, and proximal gastric folds. During the course of the endoscopy, a complete evaluation of the stomach and duodenum was carried out, and the presence of PHG and gastric varices was evaluated and recorded. Grading of esophageal varices (when present) was performed by all investigators using a predefined protocol: after examination of the stomach, the gastric cavity was fully deflated; the endoscope was withdrawn into the distal esophagus, and the esophageal lumen was fully inflated. At that point, the varices were evaluated and graded. The entire EGD was digitally recorded for reference and monitoring purposes.

\section{Ethics}

The study was conducted in accordance with the ethical principles originating from the Declaration of Helsinki, in compliance with good clinical practice, and according to local regulations. At all sites, the study protocol was approved by the local ethics committees prior to patient enrolment. All patients that enrolled in the study signed an informed consent form.

\section{Study Measurements}

Before the start of the study, each center received a kit containing representative videos of esophageal varices as seen on capsule endoscopy and instructions on how to grade them. In addition, detailed instructions on how to grade varices at EGD were given.

Grading of varices with conventional EGD employed the classification of the Italian Liver Cirrhosis Project, 5,20 in which varices are graded according to the proportion of the radius of the esophagus occupied by the largest varix present at full insufflation of the esophagus. Varices are divided into 4 grades: $\mathrm{F} 0==$ no varices; $\mathrm{F} 1==<33 \%$ of the radius of the esophagus; F2 $=33 \%-66 \%$ of the radius of the esophagus; and F3 = greater than $66 \%$ of the radius of the esophagus. The presence or absence of red spots on the varices was also noted.

Prior to the study, we also devised an esophageal variceal grading system for the video capsule endoscopy with the goal of simplicity and reproducibility for use in a multicenter study. In tackling this goal, we faced the problem of the inherent differences between EGD and videocapsule endoscopy. Standard classification of esophageal varices by EGD requires full distension of the esophagus by insufflation, but capsule endoscopy does not have insufflation capability. On the basis of the experience of the prior pilot study, ${ }^{17}$ we decided to use the circumference of the capsule picture frame as a reference point and to grade the varices according to the proportion of the circumference occupied by the largest varix present. We thus labeled as small varices occupying less than $25 \%$ of the circumference and as large varices occupying more than $25 \%$. The software for reading capsule endoscopy studies partitions the circumference of the capsule picture frame into 4 quadrants, and this partition can be rotated on the circumference of the frame. Therefore, varices were labeled as small if the largest visible varix seen in the capsule video occupied less than one quadrant and large if the largest one occupied more than that. We thus ended up with three classification grades: no varices $(\mathrm{C} 0)$, small varices (C1), and large varices (C2; Fig. 1).

\section{Patient Satisfaction Assessment}

Prior to and after completing the endoscopies, patients completed a questionnaire regarding their perceptions of the video capsule and conventional EGD. The questionnaire is shown in the appendix.

\section{Statistical Methods}

This study was designed as an equivalence study. We assumed that the prevalence of esophageal varices would be $65 \%$ and equivalent with both techniques. We also decided that a difference in the diagnosis of esophageal varices of $\leq 10 \%$ between video capsule and conventional EGD would be accepted as demonstrating equivalence between the two techniques. Therefore, in order to dem- 

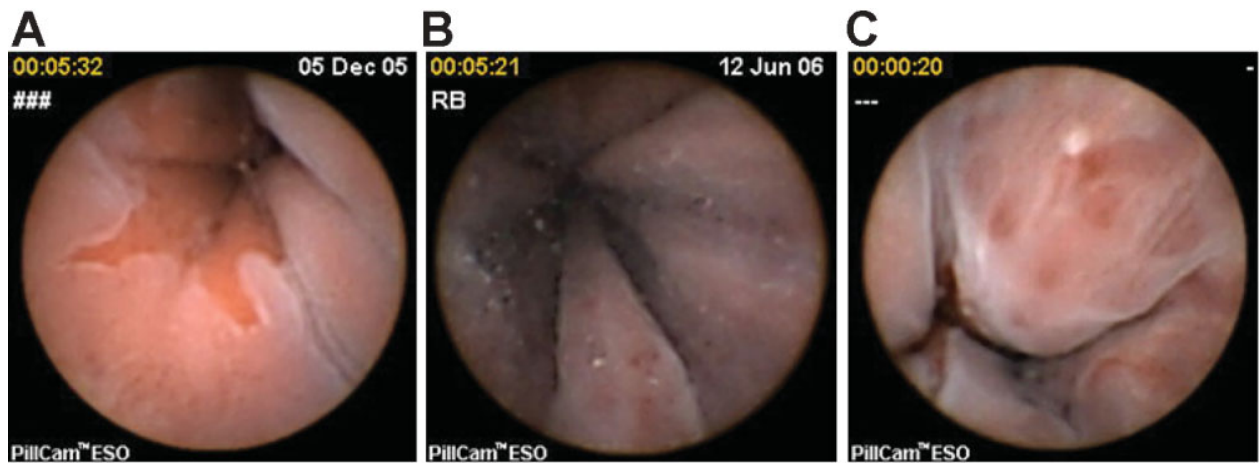

Fig. 1. Capsule endoscopy finding: (A) normal z line, no varices (C0); (B) small varices [occupying $<25 \%$ of the capsule picture frame (C1)]; and (C) large varices [occupying $>25 \%$ of the capsule picture frame (C2)].

onstrate equivalence, the boundary of the one-sided $95 \%$ confidence interval (CI) of the difference between video capsule and EGD had to be $\leq 10 \%$.

Although considerable interobserver variability exists in detecting and grading varices at EGD, ${ }^{21,22}$ we considered EGD the gold standard in all comparisons.

The required sample size for a one-tail comparison with an $\alpha$ value of 0.05 and a power of $80 \%$ is 281 patients examined with both the video capsule and EGD.

The overall agreement between the video capsule and EGD was assessed, and kappa statistics were calculated for diagnosis of varices ${ }^{23,24}$ with three different conventions: presence or absence of varices, variceal grade (none, small, or medium/large), and differentiation of medium/large varices from all other variceal grades. The last assessment was felt to be important because in clinical practice this distinction determines whether or not patients receive chronic therapy for primary prevention of variceal bleeding. The Wilcoxon test was performed in order to compare preprocedure and postprocedure mean patient satisfaction for the two procedures. $P<0.05$ was considered significant.

\section{Safety Evaluation}

One week following the esophageal capsule endoscopy procedure, patients were contacted to confirm excretion of the capsule and to verify that there were no changes in their well being following participation in this study. An $\mathrm{X}$-ray procedure was performed to confirm the capsule exit if deemed necessary by the investigator. Adverse events from study initiation to the 1-week follow-up were also recorded.

\section{Results}

Two hundred ninety patients were enrolled in the study; The number of patients enrolled at each site ranged from 10 to 51. Two patients were not included in the analysis because of loss of the capsule endoscopy recording (1) and vomiting of the capsule after ingestion due to an unsuspected esophageal stricture (1). Therefore, 290 patients were included in the safety evaluation, and 288 patients were included in the evaluation of the diagnostic yield.

The age range of the patients was 21-81 years. The baseline characteristics of the study population are summarized in Table 1. One hundred ninety-five patients had a diagnosis of portal hypertension and underwent endoscopy for the first time for screening purposes; the remaining 93 had a previous diagnosis of esophageal varices and underwent surveillance endoscopy.

\section{Efficacy Evaluation}

Comparison of Esophageal Capsule Versus EGD in the Identification of Varices (Table 2). Overall, EGD identified esophageal varices in 180 patients (62.5\%), and capsule endoscopy identified esophageal varices in 152 of these (difference 15.6\%; 95\% CI 11.4-19.8 in favor of EGD). On the other hand, in 13 cases (4.5\%), the iden-

Table 1. Demographic Data of the Whole Population

\begin{tabular}{lc}
\hline \multicolumn{1}{c}{ Gender (male/female) } & $\mathbf{1 8 1 / 1 0 5}$ \\
\hline Age [mean (range)] & 56 years (21-81) \\
Child-Pugh class & \\
A & $68.8 \%$ \\
B & $25.4 \%$ \\
C & $5.8 \%$ \\
Etiology of cirrhosis & \\
Alcohol & $20.0 \%$ \\
HBV & $8.9 \%$ \\
HCV & $35.0 \%$ \\
Alcohol + HBV or HCV cirrhosis & $13.3 \%$ \\
Other (Budd-Chiari, PVT, etc.) & $22.8 \%$ \\
Screening/surveillance & $195 / 93$ \\
\hline
\end{tabular}

HBV indicates hepatitis B virus; HCV, hepatitis C virus; and PVT, portal vein thrombosis. 
Table 2. Varices Identified by Esophageal Capsule Versus EGD

\begin{tabular}{lccc}
\hline & $\begin{array}{r}\text { Varices } \\
\text { Identified } \\
\text { by EGD }\end{array}$ & $\begin{array}{c}\text { Varices } \\
\text { Not } \\
\text { Identified } \\
\text { by EGD }\end{array}$ & Total \\
\hline Varices identified by PillCam ESO & 152 & 13 & 165 \\
Varices not identified by PillCam ESO & 28 & 95 & 123 \\
Total & 180 & 108 & 288 \\
\hline
\end{tabular}

kappa $=0.73$; sensitivity $=84 \%(\mathrm{Cl} 81 \%, 87 \%)$; specificity $=88 \%(\mathrm{Cl} 82 \%$, $92 \%)$; positive predictive value $=92 \%(\mathrm{Cl} 88 \%, 95 \%)$; negative predictive value $=77 \%(\mathrm{Cl} 72 \%, 81 \%)$; positive likelihood ratio $=7.0(\mathrm{Cl} 4.6,11.2)$ negative likelihood ratio $=0.18(\mathrm{Cl} 0.14,0.23)$.

tification of varices by the esophageal capsule was not confirmed by EGD.

The esophageal capsule and EGD findings were concordant in 247 of 288 (86\%) cases; the kappa value for the agreement between EGD and PillCam was 0.73, denoting substantial agreement. With EGD considered the gold standard, the esophageal capsule had a sensitivity of $84 \%$ and a specificity of $88 \%$ for the detection of varices. The positive and negative likelihood ratios were 7.0 and 0.18 , respectively. According to these data, the probability of having varices after a positive video capsule study rose from a pretest value of $62.5 \%$ to a posttest value of 93.0\%.

As expected in a multicenter study, the performance of the esophageal capsule in the different centers was variable; in general, the performance was better for centers enrolling large numbers of patients than for those enrolling small numbers. In four centers enrolling fewer than 15 patients, the median agreement between capsule endoscopy and EGD was $62 \%$ (range $40 \%-100 \%$ ), whereas in the 7 centers enrolling 16 patients or more, it was $92 \%$ (range 76\%-96\%).

Grading of Varices by Esophageal Capsule Versus $\boldsymbol{E G D}$. Table 3 shows the correlation between the esophageal capsule and EGD in grading esophageal varices. As judged by EGD, varices were small in 101 (35.1\%) patients and medium/large in $79(27.4 \%)$ patients. In 227 of 288 cases $(79 \%)$, there was complete agreement between the two techniques. The level of agreement measured by the kappa statistic was 0.68 , which is considered substantial agreement. Of the 28 cases $(9.7 \%)$ in which the esophageal capsule failed to detect varices identified by EGD, the missed varices were small (F1) in $24(8.3 \%)$ and medium/large (F2-F3) in $4(1.4 \%)$. In 13 cases $(4.5 \%)$, varices judged to be medium/large by EGD were considered small by the esophageal capsule. On the other hand, in 7 cases $(2.4 \%)$, varices considered small by EGD were considered large by the esophageal capsule. One of the latter patients underwent a second EGD within 5 weeks of the first one and was found to have large varices. Finally, the capsule grading in the 13 cases $(4.5 \%)$ diagnosed by the capsule but not confirmed by EGD was small in 11 and medium/large in 2.

Differentiating Large Varices from Small Varices and Absent Varices. The results of this comparison are summarized in Table 4. In 262 of 288 cases (91\%), there was complete agreement between video capsule and EGD in differentiating the two types of patients. The kappa for this comparison was 0.77 , denoting substantial agreement. The positive and negative likelihood ratios were 19.5 and 0.2, respectively. According to these figures, the likelihood of having large varices with a positive video capsule study rose from a pretest value of $27.4 \%$ to a posttest value of $85.0 \%$.

In 17 cases graded as large varices by EGD, the varices were not seen in $4(1.4 \%)$ and were graded as small in 13 (4.5\%) by capsule endoscopy. On the other hand, in 9 cases graded as large by capsule endoscopy, the varices were considered small in $7((2.4 \%)$ and absent in $2(0.7 \%)$ by EGD.

Separate Analysis of the Screening and Surveillance Populations. When the screening population (that is, the 195 patients with a diagnosis of cirrhosis who underwent endoscopy for the first time) and the surveillance population (that is, the 93 patients with a previous diagnosis of esophageal varices undergoing surveillance endoscopy) were analyzed separately, the levels of agreement between the video capsule and EGD were similar both for the detection of varices ( $83 \%$ and $91 \%$, respectively) and for the differentiation between medium/large varices and small/absent varices (91\% and $91 \%$, respectively).

Assessment of PHG. EGD identified PHG in 161 patients; capsule endoscopy identified PHG in 119 of these $(74 \%)$. On the other hand, in 21 cases $(7.3 \%)$, the identification of PHG by capsule endoscopy was not confirmed by EGD. The overall agreement between capsule endoscopy and EGD for the identification of PHG was $78 \%$ with a kappa value of 0.56 , denoting moderate agreement. With EGD considered the gold standard, the esophageal capsule had a sensitivity of $74 \%$ and a specificity of $83 \%$ for the detection of PHG. The positive and negative likelihood ratios were 4.47 and 0.31 , respectively.

\section{Safety Evaluation}

Overall, four (1.4\%) adverse events were reported within the study. One episode of severe pain occurred with EGD and improved within 1 week. The three adverse events occurring with the capsule were the following: one episode of diarrhea that resolved spontaneously within 24 hours, one episode of nausea with capsule re- 
Table 3. Grading Varices Identified by Esophageal Capsule Versus EGD in the Whole Study Population

\begin{tabular}{|c|c|c|c|c|}
\hline & $\begin{array}{l}\text { Varices Not } \\
\text { Identified } \\
\text { by EGD }\end{array}$ & $\begin{array}{c}\text { Small (F1) } \\
\text { Varices Identified } \\
\text { by EGD }\end{array}$ & $\begin{array}{c}\text { Medium/Large (F2-F3) } \\
\text { Varices Identified } \\
\text { by EGD }\end{array}$ & Total \\
\hline Varices not identified by PillCam ESO & 95 & 24 & 4 & 123 \\
\hline Small (C1) varices identified by PillCam ESO & 11 & 70 & 13 & 94 \\
\hline Medium/large (C2) varices identified by PillCam ESO & 2 & 7 & 62 & 71 \\
\hline Total & 108 & 101 & 79 & 288 \\
\hline
\end{tabular}

Overall agreement level $=79 \%(\mathrm{Cl} 74 \%, 83 \%) ;$ kappa $=0.68(P$ value $<0.0001)$.

tention due to an unsuspected esophageal stricture requiring removal of the capsule by EGD, and one episode of vomiting caused by capsule retention due to an unsuspected esophageal stricture (the capsule was passed by mouth by vomiting).

\section{Patient Satisfaction Assessment}

The results of the patient satisfaction questionnaires are summarized in Table 5. The esophageal capsule fared significantly better than EGD both for the preprocedure perception and for the postprocedure satisfaction of the patients.

\section{Discussion}

This study was designed to clarify whether esophageal capsule endoscopy is a suitable tool for the screening and surveillance of patients with cirrhosis in the detection and monitoring of esophageal varices. Its multicenter design and its size allow for a precise estimate of the diagnostic yield of this tool for the diagnosis of esophageal varices under real-life conditions.

Our comparison was designed as an equivalence test, with the assumption that a difference of $10 \%$ or less between the two techniques in the detection of esophageal varices would demonstrate equivalence of the two modalities. According to this assumption, the esophageal capsule did not reach statistical equivalence to EGD for

Table 4. Large Varices Identified by Esophageal Capsule Versus EGD

\begin{tabular}{lccc}
\hline & $\begin{array}{c}\text { Medium/Large } \\
\text { Varices Identified } \\
\text { by EGD }\end{array}$ & $\begin{array}{c}\text { Medium/Large } \\
\text { Varices Not } \\
\text { Identified by EGD }\end{array}$ & Total \\
\hline $\begin{array}{l}\text { Medium/large varices } \\
\text { identified by PillCam ESO }\end{array}$ & 62 & 9 & 71 \\
$\begin{array}{l}\text { Medium/large varices not } \\
\text { identified by PillCam ESO }\end{array}$ & 17 & 200 & 217 \\
Total & 79 & 209 & 288 \\
\hline
\end{tabular}

Overall agreement level $=91 \%(\mathrm{Cl} 87 \%-94 \%)$; kappa $=0.77(P<0.0001)$ sensitivity $=78 \%$ (95\% Cl 72\%, 83\%); specificity = 96\% (95\% Cl 93\%, 97\%); positive predictive value $=87 \%(95 \% \mathrm{Cl} 80 \%, 92 \%)$; negative predictive value $=$ $92 \%$ (95\% Cl 90\%, 94\%); positive likelihood ratio = 19.5 (95\% Cl 10.5, 32.7); negative likelihood ratio $=0.2(95 \% \mathrm{Cl} 0.17,0.3)$. diagnosing esophageal varices because the observed difference was $15.5 \%$ (95\% CI 11.4-19.8) in favor of EGD. Of the 28 cases in which the capsule failed to detect varices diagnosed by EGD, the size of the missed varices was small in $24(13.3 \%)$ and large in $4(2.2 \%)$; this indicates that the miss rate of the capsule for large varices is small.

However, the overall performance of the capsule was good: in detecting varices, the agreement between the esophageal capsule and EGD, measured by the kappa statistic, was 0.73 , which is considered substantial agreement. In addition, the positive likelihood ratio was 7.0 , and this means that the probability of having varices with a positive capsule study would increase from a pretest value of $62.5 \%$ to a posttest value of $93 \%$. The fact that the performance of capsule endoscopy was variable between centers had to be expected: the centers enrolling more patients were likely to have more experience in dealing with patients with portal hypertension and thus were more consistent in grading varices with the two methods.

As far as grading the varices is concerned, the correlation between the standard EGD classification and our tentative esophageal capsule classification was also good: the overall agreement between the two classifications was $79 \%$, with a kappa value of 0.68 , which denotes a good level of agreement. The capsule appeared to overestimate the size of varices in $7 \%$ and to underestimate it in $14 \%$ of cases. In addition, the fact that in one case, in which varices had been graded as large by the esophageal capsule and small by EGD, the varices were graded as large at repeat EGD suggests that, at least in some instances, grading by the capsule may be more precise. This is plausible because EGD grading is heavily influenced by insufflation of the esophagus.

When the two methods were compared for distinguishing between medium/large varices, which require prophylactic treatment, and small-absent varices, which require surveillance, the overall agreement was $91 \%$, with a kappa value of 0.77 , denoting substantial agreement. The positive likelihood ratio was 19.5, raising the probability of having large varices with a positive PillCam ESO 
Table 5. Preprocedure Perception and Postprocedure Satisfaction

\begin{tabular}{|c|c|c|c|}
\hline Item & $\begin{array}{l}\text { Capsule Endoscopy [Points, } \\
\text { Median (Interquartile Range)] }\end{array}$ & EGD [Points, Median (Interquartile Range)] & $P$ Value \\
\hline & \multicolumn{3}{|c|}{ Preprocedure Patient Perception } \\
\hline General anxiousness & $4(3-4)$ & $4(4-4)$ & $<0.0001$ \\
\hline \multirow[t]{2}{*}{ Fear of pain } & $3(2-4)$ & $4(3-4)$ & $<0.0001$ \\
\hline & \multicolumn{3}{|c|}{ Postprocedure Patient Satisfaction } \\
\hline Ease of swallowing/insertion & $4(3-4)$ & $3(2-4)$ & $<0.0001$ \\
\hline Pain during procedure & $4(4-4)$ & $4(3-4)$ & $<0.0001$ \\
\hline Discomfort during procedure & $4(4-4)$ & $4(3-4)$ & $<0.0001$ \\
\hline Pain after procedure & $4(4-4)$ & $4(3-4)$ & $<0.0001$ \\
\hline Discomfort after procedure & $4(4-4)$ & $4(3-4)$ & $<0.0001$ \\
\hline Examination rate & $4(3-4)$ & $3(2-3)$ & $<0.0001$ \\
\hline Selected procedure & $4(3-4)$ & $2(1-3)$ & $<0.0001$ \\
\hline Overall convenience & $3(2-3)$ & $2(1-2)$ & $<0.0001$ \\
\hline Missed time from work & $4(4-4)$ & $4(3-4)$ & $<0.0001$ \\
\hline
\end{tabular}

study from a pretest value of $27.4 \%$ to a posttest value of $85 \%$. However, by the use of the $25 \%$ threshold of the capsule picture frame to identify medium/large varices, $21.5 \%$ of patients with this type of varices identified by EGD would have been judged as having small or absent varices by the capsule and thus ineligible for primary prophylaxis. Clearly, further study is needed to clarify whether other thresholds may offer a better fit between the two classifications.

Esophageal capsule endoscopy appears to be a safe procedure because the overall incidence of adverse events occurring during the time frame of the study was $1.4 \%$. Adverse events specifically associated with the capsule occurred only in 3 patients (1\%), were mild or moderate, and resolved rapidly. The two episodes of nausea and vomiting caused by unsuspected esophageal stenoses suggest that the possible existence of stenoses, albeit rare, should be considered when an esophageal capsule study is planned as for other capsule procedures.

As expected, both the patients' perception before the procedure and satisfaction thereafter were significantly better for the esophageal capsule than for EGD.

In deciding whether the esophageal capsule is a valid alternative to EGD for the screening and surveillance of patients with portal hypertension, three issues have to be considered: performance, cost, and patients' preference. As far as performance is considered, on the basis of the primary aim of the study, EGD remains the gold standard for identifying and grading varices because the esophageal capsule, although performing well for esophageal varices, failed to reach statistical equivalence. The capsule has moderate sensitivity for diagnosing PHG and is not a suitable alternative for diagnosing gastric varices. Whether the recently proposed new ingestion procedure ${ }^{25}$ or the adoption of the new version of the esophageal capsule ${ }^{26}$ will improve performance remains to be established. As far as cost is concerned, cost analysis was beyond the scope of our study. However, a re- cently published article ${ }^{27}$ suggests that capsule screening followed by beta blocker treatment for patients with varices may be cost-effective when compared with EGD screening followed by beta blockers. Interestingly, the performance characteristics of the capsule observed in the present study fall within the performance ranges tested in the cost analysis. As far as patients' preference is concerned, EGD is perceived as unpleasant and often requires sedation, which carries some risks and delays the return of patients to work. This may decrease the adherence of patients to screening and surveillance programs. On the other hand, capsule endoscopy appears to be rated as more comfortable and convenient by patients, ${ }^{16}$ and this may lead to a better adherence to guidelines and ultimately improve the outcomes if more patients undergo screening. This will have to be determined in appropriately designed future studies.

On the basis of the results of this study, we recommend that EGD be used to screen patients with cirrhosis for large esophageal varices because capsule endoscopy, although showing good diagnostic ability, did not reach statistical equivalence to EGD for this task. Whether the use of the next-generation capsule and the new ingestion procedure can improve capsule performance will have to be ascertained by future studies. Likewise, more studies are needed to ascertain whether the simplicity and improved patient tolerance of the capsule over EGD can increase the rate of adherence to screening programs. For the time being, capsule endoscopy may be indicated for selected patients who are unwilling or unable to undergo upper gastrointestinal endoscopy.

Acknowledgment: Given Imaging provided the capsules necessary for the study.

The following participated in the study: Roberto de Franchis, M.D., Emanuele Rondonotti, M.D., Federica Villa, M.D., and Maria Grazia Rumi, M.D., Department of Medical Sciences, University of Milan, Istituto di 
Ricovero e Cura a Carattere Scientifico Fondazione Ospedale Maggiore Policlinico, Mangiagalli and Regina Elena, Milan, Italy; Glenn Eisen, M.D., Jonathan Schwartz, M.D., Douglas Faigel, M.D., and Atif Zaman, M.D., Oregon Health Sciences University, Portland, OR; Loren Laine, M.D., and Amandeep Sahuta, M.D., University of Southern California, Los Angeles, CA; Ignacio Fernandez Urién, M.D., and Miguel Muñoz Navas, M.D., Universidad de Navarra, Pamplona, Spain; Juan Manuel Herrerias, M.D., and Angel Caunedo, M.D., Hospital Universitario Virgen Macarena, Sevilla, Spain; Jay L. Goldstein, M.D., Russell D. Brown, M.D., Jamie Berkes, M.D., Scott Cotler, M.D., Allan Halline, M.D., Paul Schlesinger, M.D., and Nikunj Shah, M.D., University of Illinois at Chicago, Chicago, IL; Laurel Fisher, M.D., and Mimi Takami, M.D., University of Michigan, Ann Arbor, MI; Hugo E. Vargas, M.D., Elizabeth Carey, M.D., and Karen Wentzel, R.N., Mayo Clinic Foundation, Scottsdale, AZ; John Vargo, M.D., M.P.H., Nizar Zein, M.D., Darwin Conwell, M.D., Robert O'Shea, M.D., William Carey, M.D., and David Barnes, M.D., Cleveland Clinic Foundation, Cleveland, $\mathrm{OH}$; Coleman Smith, M.D., Julie Thompson, M.D., Robert McCabe, M.D., Robert Ganz, M.D., and Scott Ketover, M.D., Minnesota Gastroenterology, Minneapolis, MN; and Rami Eliakim, M.D., K. Yassin, M.D., E. Veitsman, M.D., and Y. Baruch, M.D., Rambam Medical Center, Haifa, Israel.

\section{Appendix: Preprocedure Patient Perception and Postprocedure Satisfaction Questionnaires Preprocedure Patient Perception}

1. How anxious are you about the PillCam ESO capsule/EGD procedure?

$4=$ not at all; $3=$ slightly; $2=$ moderately; $1=$ excessively

2. Do you expect any pain during the capsule/EGD procedure?

$4=$ not at all; $3=$ slightly; $2=$ moderately; $1=$ excessively Postprocedure Patient Satisfaction ment?

1. How would you rate the swallowing/insertion of the instru-

$4=$ very easy; $3=$ easy; $2=$ tolerable; $1=$ difficult; $0=$ very difficult

2. Did you experience pain during the procedure?

4 = none; $3=$ minor; $2=$ mild; $1=$ severe; $0=$ intolerable

3. Did you experience discomfort during the procedure?

$4=$ none; $3=$ minor; $2=$ mild; $1=$ severe; $0=$ intolerable

4. Did you experience pain after the procedure?

$4=$ none; $3=$ minor; $2=$ mild; $1=$ severe; $0=$ intolerable

5. Did you experience discomfort after the procedure?

4 = none; $3=$ minor; $2=$ mild; $1=$ severe; $0=$ intolerable

6 . How would you rate the examination procedure?

$4=$ very comfortable; $3=$ comfortable; $2=$ tolerable; $1=$ uncomfortable; $0=$ very uncomfortable

7. If you would be given the possibility to select an examination for diagnosing your problem, would you choose this particular procedure?
$4=$ definitely yes; $3=$ probably yes; $2=$ maybe; $1=$ probably not; $0=$ definitely not

8. Rate the overall convenience of the test.

3 = very convenient; $2=$ convenient; $1=$ inconvenient; $0=$ very inconvenient

9. How much time did you miss from work or regular activities due to the test? $4=0-2$ hours; $3=3-4$ hours; $2=5-6$ hours; $1=7-8$ hours; $0=>8$ hours

\section{References}

1. D’Amico G, Pagliaro L, Bosch L. Pharmacological treatment of portal hypertension: an evidence-based approach. Semin Liver Dis 1999;19:475505.

2. D'Amico G, de Franchis R, and the Cooperative Study Group. Upper digestive bleeding in cirrhosis: post-therapeutic outcomes and prognostic indicators. Hepatology 2003;38:599-612.

3. Chalasani N, Kahi C, Francois F, Pinto A, Marathe A, Bini EJ, et al. Improved patient survival after acute variceal bleeding: a multicenter, cohort study. Am J Gastroenterol 2003;98:653-659.

4. de Franchis R, Primignani M. Clin Liver Dis 2001;5:645-663.

5. North-Italian Endoscopic Club for the Study and Treatment of Esophageal Varices. Prediction of the first variceal hemorrhage in patients with cirrhosis of the liver and esophageal varices. N Engl J Med 1988;319:983989.

6. Imperiale FT, Chalasani N. A meta-analysis of endoscopic variceal ligation for primary prophylaxis of esophageal variceal bleeding. HEPATOLOGY 2001;33:802-807.

7. de Franchis R. Developing consensus in portal hypertension. J Hepatol 1996;25:390-394.

8. Grace ND, Groszmann RJ, Garcia-Tsao G, Burroughs AK, Pagliaro L, Makuch RW, et al. Portal hypertension and variceal bleeding: an AASLD single topic symposium. HePATOLOGY 1998;28:868-880.

9. Jalan R, Hayes PC. UK guidelines on the management of variceal haemorrhage in cirrhotic patients. Gut 2000;46(Suppl III):1-15.

10. de Franchis R. Updating consensus in portal hypertension. J Hepatol 2000;33:846-852.

11. D’Amico G, Garcia-Tsao G, Calés P, Escorsell A, Nevens F, Cestari R, et al. Diagnosis of portal hypertension. How and when? In: de Franchis R, ed. Portal Hypertension III. Proceedings of the Third Baveno International Consensus Workshop on Definitions, Methodology and Therapeutic Strategies. Oxford, England: Blackwell Science; 2001:3663.

12. Eisen G, Baron TH, Dominitz J, for the American Society for Gastrointestinal Endoscopy. Complications of upper GI endoscopy. Gastrointest Endosc 2002;55:784-793.

13. D’Amico G, Pasta L, Madonia S, Tarantino IG, Mancuso A, Malizia G, et al. The incidence of esophageal varices in cirrhosis. Gastroenterology 2001; 120:A2.

14. Merli M, Nicolini G, Angeloni S, Rinaldi V, De Santis A, Merkel C, et al. Incidence and natural history of small esophageal varices in cirrhotic patients. J Hepatol 2003;38:266-272.

15. de Franchis R. Noninvasive diagnosis of esophageal varices: is it feasible? Am J Gastroenterol 2006;101:2520-2522.

16. Eliakim R, Sharma VK, Yassin K, Adler SN, Jacob H, Cave DR, et al. A prospective study of the diagnostic accuracy of PillCam ESO esophageal capsule endoscopy versus conventional upper endoscopy in patients with chronic gastroesophageal reflux diseases. J Clin Gastroenterol 2005;39: 572-578.

17. Eisen GM, Eliakim R, Zaman A, Schwartz J, Faigel D, Rondonotti E, et al. The accuracy of PillCam ESO capsule endoscopy versus conventional upper endoscopy for the diagnosis of esophageal varices: a prospective threecenter pilot study. Endoscopy 2006;38:31-35.

18. Lapalus MG, Dumortier J, Fumex F, Roman S, Lot M, Prost B, et al. Esophageal capsule endoscopy versus esophagogastroduodenoscopy for evaluating portal hypertension: a prospective comparative study of performance and tolerance. Endoscopy 2006;38:36-41. 
19. Groce JR, Raju GS, Sood GK, Snyder N. A prospective single blinded comparative trial of capsule esophagoscopy vs. traditional EGD for variceal screening [Abstract]. Gastroenterology 2007;132(Suppl 2):A802.

20. The Italian Liver Cirrhosis Project. Reliability of endoscopy in the assessment of variceal features. J Hepatol 1987:4:93-98

21. Bendtsen F, Theil Skovgard L, Soerensen TIA, Matzen P. Agreement among multiple observers on endoscopic diagnosis of esophageal varices before bleeding. HePATOLOGY 1990;11:341-347.

22. Winkfield B, Aubè C, Burtin P, Calés P. Inter-observer and intra-observer variability in hepatology. Eur J Gastroenterol Hepatol 2003;15:959-966.

23. Landis JR, Koch GG. The measurement of observer agreement for categorical data. Biometrics 1977;33:159-174.
24. Brennan P, Silman A. Statistical methods for assessing observer variability in clinical measures. Br Med J 1992;304:1491-1494.

25. Gralnek IM, Rabinowitz R, Afik D, Eliakim R. A simplified ingestion procedure for esophageal capsule endoscopy: initial evaluation in healthy volunteers. Endoscopy 2006;38:913-918.

26. Gralnek IM, Adler SN, Yassin K, Koslowsky B, Metzger Y, Eliakim R. Detecting esophageal pathologies with second generation capsule endoscopy: initial evaluation of the PillCam ESO 2. Endoscopy 2007. In press.

27. Spiegel BMR, Esrailian E, Eisen G. The budget impact of endoscopic screening for esophageal varices in cirrhosis. Gastrointest Endosc 2007;66: 679-692. 